\title{
COMPARISON OF WEAK AND STRONG MOMENTS FOR VECTORS WITH INDEPENDENT COORDINATES
}

\author{
RAFAŁ LATAŁA AND MARTA STRZELECKA
}

\begin{abstract}
We show that for $p \geq 1$, the $p$-th moment of suprema of linear combinations of independent centered random variables are comparable with the sum of the first moment and the weak $p$-th moment provided that $2 q$-th and $q$-th integral moments of these variables are comparable for all $q \geq 2$. The latest condition turns out to be necessary in the i.i.d. case.
\end{abstract}

\section{Introduction and Main Results}

In many problems arising in probability theory and its applications one needs to study variables of the form $S=\sup _{t \in T}\left|\sum_{i=1}^{n} t_{i} X_{i}\right|$, where $X_{1}, \ldots, X_{n}$ are independent random variables and $T$ is a non-empty subset of $\mathbb{R}^{n}$. In particular it is of interest to estimate tails of $S$. Such estimates are strictly related to bounds for $L_{p}$-norms of $S$ (i.e. $\|S\|_{p}:=\left(\mathbb{E}|S|^{p}\right)^{1 / p}$ ) for $p \geq 1$ (see Corollary 1.3 and its proof in Section 4 below). There is a trivial lower estimate:

$$
\left(\mathbb{E} \sup _{t \in T}\left|\sum_{i=1}^{n} t_{i} X_{i}\right|^{p}\right)^{1 / p} \geq \max \left\{\mathbb{E} \sup _{t \in T}\left|\sum_{i=1}^{n} t_{i} X_{i}\right|, \sup _{t \in T}\left(\mathbb{E}\left|\sum_{i=1}^{n} t_{i} X_{i}\right|^{p}\right)^{1 / p}\right\} .
$$

It turns out that in some situations this obvious lower bound may be reversed, i.e. there exist numerical constants $C_{1}$ and $C_{2}$ such that

$$
\left(\mathbb{E} \sup _{t \in T}\left|\sum_{i=1}^{n} t_{i} X_{i}\right|^{p}\right)^{1 / p} \leq C_{1} \mathbb{E} \sup _{t \in T}\left|\sum_{i=1}^{n} t_{i} X_{i}\right|+C_{2} \sup _{t \in T}\left(\mathbb{E}\left|\sum_{i=1}^{n} t_{i} X_{i}\right|^{p}\right)^{1 / p} .
$$

This is for example the case (with $C_{1}=1$ ), when $X_{i}$ are normally distributed. This is an easy consequence of the Gaussian concentration (cf. Chapter 3 of [11). Dilworth and Montgomery-Smith [3] established the inequality (1.2) for $X_{i}$ being symmetric Bernoulli random variables. This result was generalized in [6] to symmetric variables with logarithmically concave tails and in [9. Theorem 2.3] to symmetric random variables such that $\left\|X_{i}\right\|_{q} \leq C \frac{p}{q} \alpha\left\|X_{i}\right\|_{p}$ for all $q \geq p \geq 2$.

The main result of this paper is the following.

Theorem 1.1. Let $X_{1}, \ldots, X_{n}$ be independent mean zero random variables with finite moments such that

$$
\left\|X_{i}\right\|_{2 p} \leq \alpha\left\|X_{i}\right\|_{p} \quad \text { for every } p \geq 2 \text { and } i=1, \ldots, n,
$$

where $\alpha$ is a finite positive constant. Then for every $p \geq 1$ and every non-empty set $T \subset \mathbb{R}^{n}$ we have

$$
\left(\mathbb{E} \sup _{t \in T}\left|\sum_{i=1}^{n} t_{i} X_{i}\right|^{p}\right)^{1 / p} \leq C(\alpha)\left[\mathbb{E} \sup _{t \in T}\left|\sum_{i=1}^{n} t_{i} X_{i}\right|+\sup _{t \in T}\left(\mathbb{E}\left|\sum_{i=1}^{n} t_{i} X_{i}\right|^{p}\right)^{1 / p}\right]
$$

where $C(\alpha)$ is a constant which depends only on $\alpha$.

\footnotetext{
Date: 7.12 .2016$.

The research of RL was supported by the National Science Centre, Poland grant 2015/18/A/ST1/00553 and of MS by the National Science Centre, Poland grant
} 2015/19/N/ST1/02661. 
It turns out that Theorem 1.1 may be reversed in the i.i.d. case.

Theorem 1.2. Let $X_{1}, X_{2}, \ldots$ be i.i.d. random variables. Assume that there exists a constant $L$ such that for every $p \geq 1$, every $n$ and every non-empty set $T \subset \mathbb{R}^{n}$ we have

$$
\left(\mathbb{E} \sup _{t \in T}\left|\sum_{i=1}^{n} t_{i} X_{i}\right|^{p}\right)^{1 / p} \leq L\left[\mathbb{E} \sup _{t \in T}\left|\sum_{i=1}^{n} t_{i} X_{i}\right|+\sup _{t \in T}\left(\mathbb{E}\left|\sum_{i=1}^{n} t_{i} X_{i}\right|^{p}\right)^{1 / p}\right] .
$$

Then

$$
\left\|X_{1}\right\|_{2 p} \leq \alpha(L)\left\|X_{1}\right\|_{p} \quad \text { for } p \geq 2,
$$

where $\alpha(L)$ is a constant which depends only on $L \geq 1$.

It will be clear from the proof of Theorem 1.2 that it suffices to assume (1.5) for $T=\left\{ \pm e_{j}: j \in\{1, \ldots, n\}\right\}$ only, where $\left\{e_{1}, \ldots, e_{n}\right\}$ is the canonical basis of $\mathbb{R}^{n}$.

The comparison of weak and strong moments (1.4) yields also a deviation inequality for $\sup _{t \in T}\left|\sum_{i=1}^{n} t_{i} X_{i}\right|$.

Corollary 1.3. Assume $X_{1}, X_{2}, \ldots$ satisfy the assumptions of Theorem 1.1. Then for any $u \geq 0$ and any non-empty set $T$ in $\mathbb{R}^{n}$,

$$
\begin{array}{r}
\mathbb{P}\left(\sup _{t \in T}\left|\sum_{i=1}^{n} t_{i} X_{i}\right| \geq C_{1}(\alpha)\left[u+\mathbb{E} \sup _{t \in T}\left|\sum_{i=1}^{n} t_{i} X_{i}\right|\right]\right) \\
\leq C_{2}(\alpha) \sup _{t \in T} \mathbb{P}\left(\left|\sum_{i=1}^{n} t_{i} X_{i}\right| \geq u\right)
\end{array}
$$

where constants $C_{1}(\alpha)$ and $C_{2}(\alpha)$ depend only on the constant $\alpha$ in (1.3).

Another consequence of the main theorem is the following Khintchine-Kahane type inequality.

Corollary 1.4. Assume $X_{i}, 1 \leq i \leq n$ satisfy the assumptions of Theorem 1.1. Then for any $p \geq q \geq 2$ and any non-empty set $T$ in $\mathbb{R}^{n}$ we have,

$$
\left(\mathbb{E} \sup _{t \in T}\left|\sum_{i=1}^{n} t_{i} X_{i}\right|^{p}\right)^{1 / p} \leq C_{3}(\alpha)\left(\frac{p}{q}\right)^{\max \left\{1 / 2, \log _{2} \alpha\right\}}\left(\mathbb{E} \sup _{t \in T}\left|\sum_{i=1}^{n} t_{i} X_{i}\right|^{q}\right)^{1 / q}
$$

where a constant $C_{3}(\alpha)$ depend only on the constant $\alpha$ in (1.3).

We postpone proofs of the above results and first present a number of remarks and open questions.

Remark 1.5. Exponent $\max \left\{1 / 2, \log _{2} \alpha\right\}$ in Corollary 1.4 is optimal.

Indeed, since $\|g\|_{p} \sim \sqrt{p / e}$ as $p \rightarrow \infty$ one cannot go below $1 / 2$ by the central limit theorem.

To see that $\log _{2} \alpha$ term cannot be improved it is enough to consider $\alpha>\sqrt{2}$. Let $r=1 / \log _{2} \alpha \in(0,2)$ and let $X$ be a symmetric random variable given by $\mathbb{P}(|X| \geq$ $t)=e^{-t^{r}}\left(\right.$ with $2>r>0$ ), i.e. $X=|\mathcal{E}|^{1 / r} \operatorname{sgn} \mathcal{E}$, where $\mathcal{E}$ has the symmetric exponential distribution. By Stirling's formula $\Gamma(x+1)=\left(\frac{x}{e}\right)^{x} \sqrt{2 \pi x} e^{f(x)}$ with $f(x) \in(0,1 / 12)$ for $x \geq 1$, so for $p \geq 2$,

$$
\frac{\|X\|_{2 p}}{\|X\|_{p}}=\frac{\Gamma\left(\frac{2 p}{r}+1\right)^{1 /(2 p)}}{\Gamma\left(\frac{p}{r}+1\right)^{1 / p}} \leq 2^{1 / r}\left(\frac{r}{\pi p}\right)^{1 /(4 p)} e^{1 /(24 p)} \leq 2^{1 / r}=\alpha .
$$

Moreover, $\|X\|_{p} \sim\left(\frac{p}{e r}\right)^{1 / r}$ for $p \rightarrow \infty$, so the assertion of Corollary 1.4 cannot hold with any exponent better than $\log _{2} \alpha$. 
Remark 1.6. If the variables $X_{i}$ are symmetric then the term $\mathbb{E} \sup _{t \in T}\left|\sum_{i=1}^{n} t_{i} X_{i}\right|$ in (1.4) may be replaced by $\mathbb{E} \sup _{t \in T} \sum_{i=1}^{n} t_{i} X_{i}$.

Proof. Let $s$ be any point in $T$. Then $T \subset T-T+s$, so by the triangle inequality

$$
\left(\mathbb{E} \sup _{t \in T}\left|\sum_{i=1}^{n} t_{i} X_{i}\right|^{p}\right)^{1 / p} \leq\left(\mathbb{E} \sup _{t \in T-T}\left|\sum_{i=1}^{n} t_{i} X_{i}\right|^{p}\right)^{1 / p}+\left(\mathbb{E}\left|\sum_{i=1}^{n} s_{i} X_{i}\right|^{p}\right)^{1 / p} .
$$

Estimate (1.4) applied to the set $T-T$ yields

$$
\left(\mathbb{E} \sup _{t \in T-T}\left|\sum_{i=1}^{n} t_{i} X_{i}\right|^{p}\right)^{1 / p} \leq C(\alpha)\left[\mathbb{E} \sup _{t \in T-T}\left|\sum_{i=1}^{n} t_{i} X_{i}\right|+\sup _{t \in T-T}\left(\mathbb{E}\left|\sum_{i=1}^{n} t_{i} X_{i}\right|^{p}\right)^{1 / p}\right] .
$$

The set $T-T$ is symmetric, so

$$
\mathbb{E} \sup _{t \in T-T}\left|\sum_{i=1}^{n} t_{i} X_{i}\right|=\mathbb{E} \sup _{t \in T-T} \sum_{i=1}^{n} t_{i} X_{i} \leq 2 \mathbb{E} \sup _{t \in T} \sum_{i=1}^{n} t_{i} X_{i}
$$

where the last estimate follows, since $\left(X_{i}\right)_{i=1}^{n}$ and $\left(-X_{i}\right)_{i=1}^{n}$ are equally distributed. Moreover,

$$
\sup _{t \in T-T}\left(\mathbb{E}\left|\sum_{i=1}^{n} t_{i} X_{i}\right|^{p}\right)^{1 / p} \leq 2 \sup _{t \in T}\left(\mathbb{E}\left|\sum_{i=1}^{n} t_{i} X_{i}\right|^{p}\right)^{1 / p},
$$

what finishes the proof of the remark.

Remark 1.7. If the variables $X_{i}$ are not centered then (1.4) holds provided that the assumption (1.3) is replaced by

$$
\left\|X_{i}-\mathbb{E} X_{i}\right\|_{2 p} \leq \alpha\left\|X_{i}-\mathbb{E} X_{i}\right\|_{p} \quad \text { for } p \geq 2 \text { and } i=1, \ldots, n .
$$

Proof. We have

$$
\left(\mathbb{E} \sup _{t \in T}\left|\sum_{i=1}^{n} t_{i} X_{i}\right|^{p}\right)^{1 / p} \leq\left(\mathbb{E} \sup _{t \in T}\left|\sum_{i=1}^{n} t_{i}\left(X_{i}-\mathbb{E} X_{i}\right)\right|^{p}\right)^{1 / p}+\sup _{t \in T}\left|\sum_{i=1}^{n} t_{i} \mathbb{E} X_{i}\right| .
$$

Theorem 1.1 applied to centered variables $X_{i}-\mathbb{E} X_{i}, i=1, \ldots, n$, yields

$$
\begin{aligned}
& \left(\mathbb{E} \sup _{t \in T}\left|\sum_{i=1}^{n} t_{i}\left(X_{i}-\mathbb{E} X_{i}\right)\right|^{p}\right)^{1 / p} \\
& \quad \leq C(\alpha)\left[\mathbb{E} \sup _{t \in T}\left|\sum_{i=1}^{n} t_{i}\left(X_{i}-\mathbb{E} X_{i}\right)\right|+\sup _{t \in T}\left(\mathbb{E}\left|\sum_{i=1}^{n} t_{i}\left(X_{i}-\mathbb{E} X_{i}\right)\right|^{p}\right)^{1 / p}\right] .
\end{aligned}
$$

To conclude it is enough to observe that

$$
\begin{gathered}
\mathbb{E} \sup _{t \in T}\left|\sum_{i=1}^{n} t_{i}\left(X_{i}-\mathbb{E} X_{i}\right)\right| \leq \mathbb{E} \sup _{t \in T}\left|\sum_{i=1}^{n} t_{i} X_{i}\right|+\sup _{t \in T}\left|\sum_{i=1}^{n} t_{i} \mathbb{E} X_{i}\right|, \\
\sup _{t \in T}\left(\mathbb{E}\left|\sum_{i=1}^{n} t_{i}\left(X_{i}-\mathbb{E} X_{i}\right)\right|^{p}\right)^{1 / p} \leq \sup _{t \in T}\left(\mathbb{E}\left|\sum_{i=1}^{n} t_{i} X_{i}\right|^{p}\right)^{1 / p}+\sup _{t \in T}\left|\sum_{i=1}^{n} t_{i} \mathbb{E} X_{i}\right|,
\end{gathered}
$$

and

$$
\sup _{t \in T}\left|\sum_{i=1}^{n} t_{i} \mathbb{E} X_{i}\right| \leq \mathbb{E} \sup _{t \in T}\left|\sum_{i=1}^{n} t_{i} X_{i}\right|
$$


Open questions. For Gaussian random vectors (1.2) holds with $C_{1}=1$. This is also the case for $X_{i}$ symmetric, independent with log-concave distributions [10, Remark 3.16 and Corollary 2.19]. However, we do not know the general conditions for the distributions of $X_{i}$ which are sufficient for (1.2) to hold with $C_{1}=1$.

It is of interest to study the comparison of weak and strong moments for random vectors $X=\left(X_{1}, \ldots, X_{n}\right)$ with dependent coordinates. A natural and important class to investigate in this context are vectors with log-concave distributions (cf. 2] for an up to date survey of properties of such vectors). Paouris [12] showed that (1.2) holds for log-concave vectors and sets $T$ being balls in Euclidean spaces (see also [1). This was generalized in 8 to balls in $L_{r}$-spaces with $1 \leq r<\infty$. Unfortunately there are very few classes of log-concave vectors such that (1.2) is known to be satisfied for all sets $T$ - this includes vectors uniformly distributed on $l_{r}^{n}$-balls (with $1 \leq r \leq \infty$ ). [10, Remark 3.16 and Theorem 5.27], or more generally vectors with densities of the form $\exp \left(-\varphi\left(\|x\|_{r}\right)\right)$, where $\varphi:[0, \infty) \rightarrow(-\infty, \infty]$ is non-decreasing and convex, and $1 \leq r \leq \infty$ [7, Proposition 6.5].

The organization of this paper is as follows. In Section 2 we prove Theorem 1.1 for unconditional sets $T$ only. Using this result we generalize it to the case of an arbitrary $T$ in Section 3. In Section 4 we prove Corollaries 1.3 and 1.4 Finally, in Section 5 we present the proof of Theorem 1.2

Throughout this paper by a letter $C$ we denote universal constants and by $C(\alpha)$ constants depending only on the parameter $\alpha$. The values of the constants $C$, $C(\alpha)$ may differ at each occurrence. We will also frequently work with a Bernoulli sequence $\varepsilon_{i}$ of i.i.d. symmetric random variables taking values \pm 1 . We assume that variables $\varepsilon_{i}$ are independent of other random variables.

\section{The CASE OF UnCONDITIONAL SETS}

In this section we show that Theorem 1.1 holds under additional assumptions that the set $T$ is unconditional and the variables $X_{i}$ are symmetric. Recall that a set $T$ in $\mathbb{R}^{n}$ is called unconditional if it is symmetric with respect to the coordinate axes, i.e. $\left(\eta_{i} t_{i}\right)_{i=1}^{n} \in T$ for any $t=\left(t_{i}\right)_{i=1}^{n} \in T$ and any choice of signs $\eta_{1}, \ldots, \eta_{n} \in$ $\{-1,1\}$.

Proposition 2.1. Let $r \in(0,1)$ and $L \geq 1$. Assume that variables $Y_{1}, \ldots, Y_{n}$ are independent and symmetric and

$$
\left(\mathbb{E} \sup _{t \in T}\left|\sum_{i=1}^{n} t_{i} Y_{i}\right|^{p}\right)^{1 / p} \leq L\left[\mathbb{E} \sup _{t \in T} \sum_{i=1}^{n} t_{i} Y_{i}+\sup _{t \in T}\left(\mathbb{E}\left|\sum_{i=1}^{n} t_{i} Y_{i}\right|^{p}\right)^{1 / p}\right]
$$

for all $p \geq 1$ and all unconditional sets $T$. Then variables $X_{i}:=\left|Y_{i}\right|^{1 / r} \operatorname{sgn} Y_{i}$ satisfy

$$
\left(\mathbb{E} \sup _{t \in T}\left|\sum_{i=1}^{n} t_{i} X_{i}\right|^{p}\right)^{1 / p} \leq(2 L)^{1 / r}\left[\mathbb{E} \sup _{t \in T} \sum_{i=1}^{n} t_{i} X_{i}+\sup _{t \in T}\left(\mathbb{E}\left|\sum_{i=1}^{n} t_{i} X_{i}\right|^{p}\right)^{1 / p}\right]
$$

for all $p \geq 1$ and all unconditional sets $T \subset \mathbb{R}^{n}$.

Proof. Definition of $X_{i}$ and unconditionality of $T$ yield

$$
\sup _{t \in T}\left|\sum_{i=1}^{n} t_{i} X_{i}\right|=\left.\left.\sup _{t \in T}\left|\sum_{i=1}^{n} t_{i}\right| Y_{i}\right|^{1 / r} \operatorname{sgn} Y_{i}\left|=\sup _{t \in T} \sum_{i=1}^{n}\right| t_{i}|| Y_{i}\right|^{1 / r} .
$$

Let $s=(1-r)^{-1}$ and let $B_{s}^{n}$ denote the unit ball of $\ell_{s}^{n}$. Then $1 / s+r=1$ and by Hölder's duality we have

$$
\left.\left.\sup _{t \in T}\left|\sum_{i=1}^{n}\right| t_{i}|| Y_{i}\right|^{1 / r}\right|^{r}=\sup _{t \in T} \sup _{u \in B_{s}^{n}} \sum_{i=1}^{n} u_{i}\left|t_{i}\right|^{r} Y_{i}=\sup _{t \in T_{r}} \sum_{i=1}^{n} t_{i} Y_{i}
$$


where

$$
T_{r}:=\left\{\left(u_{i}\left|t_{i}\right|^{r}\right)_{i=1}^{n}: t \in T, u \in B_{s}^{n}\right\}
$$

is unconditional in $\mathbb{R}^{n}$. Therefore (2.1) applied with $p / r$ and $T_{r}$ instead of $p$ and $T$ yields

$$
\mathbb{E} \sup _{t \in T}\left|\sum_{i=1}^{n} t_{i} X_{i}\right|^{p} \leq L^{p / r}\left[\mathbb{E} \sup _{t \in T_{r}} t_{i} Y_{i}+\sup _{t \in T_{r}}\left(\mathbb{E}\left|\sum_{i=1}^{n} t_{i} Y_{i}\right|^{p / r}\right)^{r / p}\right]^{p / r} .
$$

We have

Moreover,

$$
\mathbb{E} \sup _{t \in T_{r}} \sum_{i=1}^{n} t_{i} Y_{i}=\mathbb{E} \sup _{t \in T}\left|\sum_{i=1}^{n} t_{i} X_{i}\right|^{r} \leq\left(\mathbb{E} \sup _{t \in T}\left|\sum_{i=1}^{n} t_{i} X_{i}\right|\right)^{r}
$$

$$
\begin{aligned}
\sup _{t \in T_{r}}\left(\mathbb{E}\left|\sum_{i=1}^{n} t_{i} Y_{i}\right|^{p / r}\right)^{r / p} & \leq \sup _{t \in T}\left(\left.\left.\mathbb{E} \sup _{u \in B_{s}^{n}}\left|\sum_{i=1}^{n} u_{i}\right| t_{i}\right|^{r} Y_{i}\right|^{p / r}\right)^{r / p} \\
& =\sup _{t \in T}\left(\mathbb{E}\left|\sum_{i=1}^{n}\right| t_{i}|| X_{i}||^{p}\right)^{r / p}=\sup _{t \in T}\left(\mathbb{E}\left|\sum_{i=1}^{n} t_{i}\right| X_{i}||^{p}\right)^{r / p} .
\end{aligned}
$$

Estimates above together with the inequality $(a+b)^{1 / r} \leq 2^{1 / r-1}\left(a^{1 / r}+b^{1 / r}\right)$ yield

$$
\left(\mathbb{E} \sup _{t \in T}\left|\sum_{i=1}^{n} t_{i} X_{i}\right|^{p}\right)^{1 / p} \leq \frac{1}{2}(2 L)^{1 / r}\left[\mathbb{E} \sup _{t \in T}\left|\sum_{i=1}^{n} t_{i} X_{i}\right|+\sup _{t \in T}\left(\mathbb{E}\left|\sum_{i=1}^{n} t_{i}\right| X_{i}||^{p}\right)^{1 / p}\right] .
$$

Hence, in order to prove (2.2) it suffices to show that

$$
\sup _{t \in T}\left(\mathbb{E}\left|\sum_{i=1}^{n} t_{i}\right| X_{i}||^{p}\right)^{1 / p} \leq \mathbb{E} \sup _{t \in T} \sum_{i=1}^{n} t_{i} X_{i}+2 \sup _{t \in T}\left(\mathbb{E}\left|\sum_{i=1}^{n} t_{i} X_{i}\right|^{p}\right)^{1 / p} .
$$

Let $\left(X_{1}^{\prime}, \ldots, X_{n}^{\prime}\right)$ be an independent copy of $\left(X_{1}, \ldots, X_{n}\right)$. By the triangle inequality for the $p$-th integral norm and Jensen's inequality we get

$$
\begin{aligned}
\sup _{t \in T}\left(\mathbb{E}\left|\sum_{i=1}^{n} t_{i}\right| X_{i}||^{p}\right)^{1 / p} & \leq \sup _{t \in T}\left(\mathbb{E}\left|\sum_{i=1}^{n} t_{i}\left(\left|X_{i}\right|-\mathbb{E}\left|X_{i}^{\prime}\right|\right)\right|^{p}\right)^{1 / p}+\sup _{t \in T}\left|\mathbb{E} \sum_{i=1}^{n} t_{i}\right| X_{i}|| \\
& \leq \sup _{t \in T}\left(\mathbb{E}\left|\sum_{i=1}^{n} t_{i}\left(\left|X_{i}\right|-\left|X_{i}^{\prime}\right|\right)\right|^{p}\right)^{1 / p}+\mathbb{E} \sup _{t \in T}\left|\sum_{i=1}^{n} t_{i}\right| X_{i}|| \\
& =\sup _{t \in T}\left(\mathbb{E}\left|\sum_{i=1}^{n} t_{i}\left(\left|X_{i}\right|-\left|X_{i}^{\prime}\right|\right)\right|^{p}\right)^{1 / p}+\mathbb{E} \sup _{t \in T} \sum_{i=1}^{n} t_{i} X_{i},
\end{aligned}
$$

where the equation follows by the unconditionality of $T$.

Let $\left(\varepsilon_{i}\right)_{i=1}^{n}$ be the Bernoulli sequence, independent of all $X_{i}$ and $X_{i}^{\prime}$. Then the sequence $\left(\left|X_{i}\right|-\left|X_{i}^{\prime}\right|\right)_{i=1}^{n}$ has the same distribution as $\left(\varepsilon_{i}\left(\left|X_{i}\right|-\left|X_{i}^{\prime}\right|\right)\right)_{i=1}^{n}$ and for every $t \in \mathbb{R}^{n}$,

$$
\begin{aligned}
\left(\mathbb{E}\left|\sum_{i=1}^{n} t_{i}\left(\left|X_{i}\right|-\left|X_{i}^{\prime}\right|\right)\right|^{p}\right)^{1 / p} & =\left(\mathbb{E}\left|\sum_{i=1}^{n} t_{i} \varepsilon_{i}\left(\left|X_{i}\right|-\left|X_{i}^{\prime}\right|\right)\right|^{p}\right)^{1 / p} \\
& \leq\left(\mathbb{E}\left|\sum_{i=1}^{n} t_{i} \varepsilon_{i}\right| X_{i}||^{p}\right)^{1 / p}+\left(\mathbb{E}\left|\sum_{i=1}^{n} t_{i} \varepsilon_{i}\right| X_{i}^{\prime}||^{p}\right)^{1 / p} \\
& =2\left(\mathbb{E}\left|\sum_{i=1}^{n} t_{i} X_{i}\right|^{p}\right)^{1 / p} .
\end{aligned}
$$

Putting (2.4) and (2.5) together we get (2.3), what completes the proof of (2.2). 
Corollary 2.2. Let $X_{1}, \ldots, X_{n}$ be independent symmetric random variables with finite moments such that

$$
\left\|X_{i}\right\|_{2 p} \leq \alpha\left\|X_{i}\right\|_{p} \quad \text { for } p \geq 2 \text { and } i=1, \ldots, n,
$$

where $\alpha$ is a finite positive constant. Then for every $p \geq 1$ and every unconditional set $T \subset \mathbb{R}^{n}$ we have

$$
\left(\mathbb{E} \sup _{t \in T}\left|\sum_{i=1}^{n} t_{i} X_{i}\right|^{p}\right)^{1 / p} \leq C(\alpha)\left[\mathbb{E} \sup _{t \in T} \sum_{i=1}^{n} t_{i} X_{i}+\sup _{t \in T}\left(\mathbb{E}\left|\sum_{i=1}^{n} t_{i} X_{i}\right|^{p}\right)^{1 / p}\right],
$$

where $C(\alpha)$ is a constant, which depends only on $\alpha$.

Proof. Let us first note, that the assumption (2.6) applied $k$ times yields that

$$
\left\|X_{i}\right\|_{2^{k} p} \leq \alpha^{k}\left\|X_{i}\right\|_{p} \quad \text { for } p \geq 2 \text {. }
$$

Therefore

$$
\left\|X_{i}\right\|_{q} \leq \alpha^{\left\lceil\log _{2}\left(\frac{q}{p}\right)\right\rceil}\left\|X_{i}\right\|_{p} \leq \alpha\left(\frac{q}{p}\right)^{\log _{2} \alpha}\left\|X_{i}\right\|_{p} \quad \text { for } q \geq p \geq 2 .
$$

Let $Y_{i}:=\left|X_{i}\right|^{1 / \log _{2} \alpha} \operatorname{sgn} X_{i}$. Then $X_{i}=\left|Y_{i}\right|^{1 / r} \operatorname{sgn} Y_{i}$ with $r:=\frac{1}{\log _{2} \alpha}$ and

$$
\left\|Y_{i}\right\|_{q} \leq 2 \frac{q}{p}\left\|Y_{i}\right\|_{p} \quad \text { for } q \geq p \geq 2 \log _{2} \alpha .
$$

If $\alpha \leq 2$ we have

$$
\left\|Y_{i}\right\|_{q} \leq 2 \frac{q}{p}\left\|Y_{i}\right\|_{p} \quad \text { for } q \geq p \geq 2 .
$$

Otherwise, take $2 \log _{2} \alpha \geq q \geq p \geq 2$. Then by Hölder's inequality and (2.8) with exponents $\frac{p(q-1)}{p-1}$ and $q$ we get

$$
\left\|Y_{i}\right\|_{q}^{q}=\mathbb{E}\left|Y_{i}\right|\left|Y_{i}\right|^{q-1} \leq\left(\mathbb{E}\left|Y_{i}\right|^{p}\right)^{\frac{1}{p}}\left(\mathbb{E}\left|Y_{i}\right|^{\frac{p(q-1)}{p-1}}\right)^{\frac{p-1}{p}} \leq\left\|Y_{i}\right\|_{p}\left\|Y_{i}\right\|_{q}^{q-1}\left(2 \frac{p(q-1)}{q(p-1)}\right)^{q-1} .
$$

Observe that

So

$$
\left(2 \frac{p(q-1)}{q(p-1)}\right)^{q-1} \leq 4^{q-1} \leq \frac{1}{4} \alpha^{4}
$$

$$
\left\|Y_{i}\right\|_{q} \leq \frac{1}{4} \alpha^{4}\left\|Y_{i}\right\|_{p} \quad \text { for } 2 \log _{2} \alpha \geq q \geq p \geq 2 .
$$

Thus for any value of $\alpha$ we get

$$
\left\|Y_{i}\right\|_{q} \leq \max \left\{2, \frac{1}{2} \alpha^{4}\right\} \frac{q}{p}\left\|Y_{i}\right\|_{p} \quad \text { for } q \geq p \geq 2 .
$$

Hence, by [9, Theorem 2.3] the variables $Y_{1}, \ldots, Y_{n}$ satisfy (2.1) (in fact for arbitrary, not only unconditional sets $T$ ) and the assertion follows by Proposition 2.1

\section{Symmetrization ARgument}

We will use the following proposition to prove that we may skip the unconditionality assumption in Corollary 2.2

Proposition 3.1. Let $\left(X_{i}\right)_{i=1}^{n}$ be a sequence of independent random variables with finite second moments and let $\left(\varepsilon_{i}\right)_{i=1}^{n}$ be a Bernoulli sequence independent of $\left(X_{i}\right)_{i=1}^{n}$. Then for any $T \subset \mathbb{R}^{n}$ and $p \geq 1$,

$$
\mathbb{E}_{X} \sup _{t \in T}\left(\mathbb{E}_{\varepsilon}\left|\sum_{i=1}^{n} t_{i} \varepsilon_{i} X_{i}\right|^{p}\right)^{1 / p} \leq C\left[\mathbb{E} \sup _{t \in T} \sum_{i=1}^{n} t_{i} \varepsilon_{i} X_{i}+\sup _{t \in T}\left(\mathbb{E}\left|\sum_{i=1}^{n} t_{i} \varepsilon_{i} X_{i}\right|^{p}\right)^{1 / p}\right] .
$$


Proof. Since this is only a matter of normalization we may and will assume that $\mathbb{E} X_{i}^{2}=1$ for all $i$.

Let $m$ be such an integer that $2 m \leq p<2(m+1)$. Then, by the symmetry of $X_{i}, \varepsilon_{i}$, and the independence of $X_{1}, \ldots, X_{n}, \varepsilon_{1}, \ldots, \varepsilon_{n}$ we have

$$
\begin{aligned}
\left\|\sum_{i=1}^{n} t_{i} \varepsilon_{i} X_{i}\right\|_{p} & \geq\left\|\sum_{i=1}^{n} t_{i} \varepsilon_{i} X_{i}\right\|_{2 m} \\
& =\left(\sum_{i_{1}+\ldots i_{n}=m} c_{i_{1}, \ldots, i_{n}} t_{1}^{2 i_{1}} \ldots t_{n}^{2 i_{n}} \mathbb{E} X_{1}^{2 i_{1}} \ldots \mathbb{E} X_{n}^{2 i_{n}}\right)^{1 / 2 m} \\
& \geq\left(\sum_{i_{1}+\ldots i_{n}=m} c_{i_{1}, \ldots, i_{n}} t_{1}^{2 i_{1}} \ldots t_{n}^{2 i_{n}}\right)^{1 / 2 m} \\
& =\left(\sum_{i_{1}+\ldots i_{n}=m} c_{i_{1}, \ldots, i_{n}} t_{1}^{2 i_{1}} \ldots t_{n}^{2 i_{n}} \mathbb{E} \varepsilon_{1}^{2 i_{1}} \ldots \mathbb{E} \varepsilon_{n}^{2 i_{n}}\right)^{1 / 2 m}=\left\|\sum_{i=1}^{n} t_{i} \varepsilon_{i}\right\|_{2 m},
\end{aligned}
$$

where

$$
c_{i_{1}, \ldots, i_{n}}=\frac{\left(2 i_{1}+\ldots+2 i_{n}\right) !}{\left(2 i_{1}\right) ! \ldots\left(2 i_{n}\right) !} .
$$

Moreover by the result of Hitczenko [4,

$$
\left\|\sum_{i=1}^{n} t_{i} \varepsilon_{i}\right\|_{2 m} \geq \frac{1}{C}\left[\sum_{i \leq 2 m} t_{i}^{*}+\sqrt{2 m} \sqrt{\sum_{i>2 m}\left|t_{i}^{*}\right|^{2}}\right],
$$

where $\left(t_{i}^{*}\right)_{i=1}^{n}$ denotes the non-increasing rearrangement of $\left(\left|t_{i}\right|\right)_{i=1}^{n}$.

Therefore to establish (3.1) it is enough to show that

$$
\mathbb{E} \sup _{t \in T}\left(\mathbb{E}_{\varepsilon}\left|\sum_{i=1}^{n} t_{i} \varepsilon_{i} X_{i}\right|^{p}\right)^{1 / p} \leq C\left(\mathbb{E} \sup _{t \in T} \sum_{i=1}^{n} t_{i} \varepsilon_{i} X_{i}+p a\right),
$$

where

$$
a:=\frac{1}{p} \sup _{t \in T}\left(\sum_{i \leq p} t_{i}^{*}+\sqrt{p}\left(\sum_{i>p}\left|t_{i}^{*}\right|^{2}\right)^{1 / 2}\right) .
$$

To this end observe that since

$$
\begin{gathered}
\left\|\sum_{i=1}^{n} u_{i} \varepsilon_{i}\right\|_{p} \leq C \sqrt{p}\|u\|_{2}, \quad\left\|\sum_{i=1}^{n} u_{i} \varepsilon_{i}\right\|_{p} \leq\|u\|_{1}, \\
\text { and }\left\|\sum_{i=1}^{n} u_{i} \varepsilon_{i}\right\|_{p}=\left\|\sum_{i=1}^{n}\left|u_{i}\right| \varepsilon_{i}\right\|_{p},
\end{gathered}
$$

we have

$$
\left\|\sum_{i=1}^{n} u_{i} \varepsilon_{i}\right\|_{p} \leq \sum_{i=1}^{n}\left(\left|u_{i}\right|-a\right)_{+}+C \sqrt{p}\left(\sum_{i=1}^{n} \min \left\{u_{i}^{2}, a^{2}\right\}\right)^{1 / 2}
$$

Thus

$$
\begin{aligned}
& \mathbb{E}_{X} \sup _{t \in T}\left(\mathbb{E}_{\varepsilon}\left|\sum_{i=1}^{n} t_{i} \varepsilon_{i} X_{i}\right|^{p}\right)^{1 / p} \\
& \quad \leq \mathbb{E} \sup _{t \in T} \sum_{i=1}^{n}\left(\left|t_{i} X_{i}\right|-a\right)_{+}+C \sqrt{p}\left(\mathbb{E} \sup _{t \in T} \sum_{i=1}^{n} \min \left\{\left(t_{i} X_{i}\right)^{2}, a^{2}\right\}\right)^{1 / 2} .
\end{aligned}
$$


To estimate the first term above observe that

$$
\begin{aligned}
& \mathbb{E} \sup _{t \in T} \sum_{i=1}^{n}\left(\left|t_{i} X_{i}\right|-a\right)_{+} \\
& \quad \leq \sup _{t \in T} \mathbb{E} \sum_{i=1}^{n}\left(\left|t_{i} X_{i}\right|-a\right)_{+}+\mathbb{E} \sup _{t \in T} \sum_{i=1}^{n}\left(\left(\left|t_{i} X_{i}\right|-a\right)_{+}-\mathbb{E}\left(\left|t_{i} X_{i}^{\prime}\right|-a\right)_{+}\right),
\end{aligned}
$$

where $\left(X_{i}^{\prime}\right)_{i}$ is a copy of $\left(X_{i}\right)$, independent of $\left(\varepsilon_{i}\right)$ and $\left(X_{i}\right)$.

Observe that for any $u$ and $i$

$$
\mathbb{E}\left(\left|u X_{i}\right|-a\right)_{+} \leq|u| \mathbb{E}\left|X_{i}\right| \leq|u|\left\|X_{i}\right\|_{2}=|u|
$$

and, by the Cauchy-Schwarz inequality and the Markov inequality

$$
\begin{aligned}
\mathbb{E}\left(\left|u X_{i}\right|-a\right)_{+} & \leq|u| \mathbb{E}\left|X_{i}\right| I_{\left\{\left|X_{i}\right| \geq a /|u|\right\}} \leq|u|\left\|X_{i}\right\|_{2}\left(\mathbb{P}\left(\left|X_{i}\right| \geq a /|u|\right)\right)^{1 / 2} \\
& \leq|u|\left\|X_{i}\right\|_{2}^{2} \frac{|u|}{a}=\frac{u^{2}}{a} .
\end{aligned}
$$

Hence for any $t \in T$

$$
\sum_{i=1}^{n} \mathbb{E}\left(\left|t_{i} X_{i}\right|-a\right)_{+} \leq \sum_{i \leq p} t_{i}^{*}+\frac{1}{a} \sum_{i>p}\left(t_{i}^{*}\right)^{2} \leq 2 p a .
$$

Moreover, by the Jensen inequality

$$
\begin{aligned}
\mathbb{E} \sup _{t \in T} \sum_{i=1}^{n}\left(\left(\left|t_{i} X_{i}\right|-a\right)_{+}\right. & \left.-\mathbb{E}\left(\left|t_{i} X_{i}^{\prime}\right|-a\right)_{+}\right) \\
& \leq \mathbb{E} \sup _{t \in T} \sum_{i=1}^{n}\left(\left(\left|t_{i} X_{i}\right|-a\right)_{+}-\left(\left|t_{i} X_{i}^{\prime}\right|-a\right)_{+}\right) \\
& =\mathbb{E} \sup _{t \in T} \sum_{i=1}^{n} \varepsilon_{i}\left(\left(\left|t_{i} X_{i}\right|-a\right)_{+}-\left(\left|t_{i} X_{i}^{\prime}\right|-a\right)_{+}\right) \\
& \leq \mathbb{E} \sup _{t \in T} \sum_{i=1}^{n} \varepsilon_{i}\left(\left|t_{i} X_{i}\right|-a\right)_{+}+\mathbb{E} \sup _{t \in T} \sum_{i=1}^{n}-\varepsilon_{i}\left(\left|t_{i} X_{i}^{\prime}\right|-a\right)_{+} \\
& =2 \mathbb{E} \sup _{t \in T} \sum_{i=1}^{n} \varepsilon_{i}\left(\left|t_{i} X_{i}\right|-a\right)_{+} \cdot
\end{aligned}
$$

Function $x \mapsto(|x|-a)_{+}$is 1-Lipschitz, so Talagrand's comparison theorem for Bernoulli processes [13, Theorem 2.1] yields

$$
\mathbb{E} \sup _{t \in T} \sum_{i=1}^{n} \varepsilon_{i}\left(\left|t_{i} X_{i}\right|-a\right)_{+} \leq \mathbb{E} \sup _{t \in T} \sum_{i=1}^{n} t_{i} \varepsilon_{i} X_{i} .
$$

Therefore

$$
\mathbb{E} \sup _{t \in T} \sum_{i=1}^{n}\left(\left|t_{i} X_{i}\right|-a\right)_{+} \leq 2 p a+2 \mathbb{E} \sup _{t \in T} \sum_{i=1}^{n} t_{i} \varepsilon_{i} X_{i} .
$$

Now we turn our attention to the other term in (3.3). We have

$$
\begin{aligned}
& \mathbb{E} \sup _{t \in T} \sum_{i=1}^{n} \min \left\{\left(t_{i} X_{i}\right)^{2}, a^{2}\right\} \\
& \leq \sup _{t \in T} \mathbb{E} \sum_{i=1}^{n} \min \left\{\left(t_{i} X_{i}\right)^{2}, a^{2}\right\}+\mathbb{E} \sup _{t \in T} \sum_{i=1}^{n}\left(\min \left\{\left(t_{i} X_{i}\right)^{2}, a^{2}\right\}-\mathbb{E} \min \left\{\left(t_{i} X_{i}\right)^{2}, a^{2}\right\}\right) .
\end{aligned}
$$


We have

$$
\sum_{i=1}^{n} \mathbb{E} \min \left\{\left(t_{i} X_{i}\right)^{2}, a^{2}\right\} \leq \sum_{i=1}^{n} \min \left\{a^{2}, t_{i}^{2} \mathbb{E} X_{i}^{2}\right\} \leq p a^{2}+\sum_{i>p}\left(t_{i}^{*}\right)^{2} \leq 2 p a^{2} .
$$

Moreover, by the Jensen inequality

$$
\begin{aligned}
\mathbb{E} \sup _{t \in T} \sum_{i=1}^{n}\left(\min \left\{\left(t_{i} X_{i}\right)^{2}, a^{2}\right\}-\right. & \left.\mathbb{E} \min \left\{\left(t_{i} X_{i}^{\prime}\right)^{2}, a^{2}\right\}\right) \\
& \leq \mathbb{E} \sup _{t \in T} \sum_{i=1}^{n}\left(\min \left\{\left(t_{i} X_{i}\right)^{2}, a^{2}\right\}-\min \left\{\left(t_{i} X_{i}^{\prime}\right)^{2}, a^{2}\right\}\right) \\
& =\mathbb{E} \sup _{t \in T} \sum_{i=1}^{n} \varepsilon_{i}\left(\min \left\{\left(t_{i} X_{i}\right)^{2}, a^{2}\right\}-\min \left\{\left(t_{i} X_{i}^{\prime}\right)^{2}, a^{2}\right\}\right) \\
& \leq 2 \mathbb{E} \sup _{t \in T} \sum_{i=1}^{n} \varepsilon_{i} \min \left\{\left(t_{i} X_{i}\right)^{2}, a^{2}\right\} .
\end{aligned}
$$

Function $x \mapsto \min \left\{x^{2}, a^{2}\right\}$ is $2 a$-Lipschitz, so using the comparison theorem for Bernoulli processes again we get

$$
\mathbb{E} \sup _{t \in T} \sum_{i=1}^{n} \varepsilon_{i} \min \left\{\left(t_{i} X_{i}\right)^{2}, a^{2}\right\} \leq 2 a \mathbb{E} \sup _{t \in T} \sum_{i=1}^{n} t_{i} \varepsilon_{i} X_{i} .
$$

Thus

$$
\begin{aligned}
p \mathbb{E} \sup _{t \in T} \sum_{i=1}^{n} \min \left\{\left(t_{i} X_{i}\right)^{2}, a^{2}\right\} \leq 2 p^{2} a^{2}+4 p a \mathbb{E} \sup _{t \in T} \sum_{i=1}^{n} t_{i} \varepsilon_{i} X_{i} & \\
& \leq\left(2 p a+\mathbb{E} \sup _{t \in T} \sum_{i=1}^{n} t_{i} \varepsilon_{i} X_{i}\right)^{2} .
\end{aligned}
$$

Estimate (3.2) follows by (3.3)- (3.5)

Proof of Theorem 1.1. Since it is enough to consider $T \cup(-T)$ instead of $T$, we may and will assume that the set $T$ is symmetric, i.e. $T=-T$.

Assume first that the variables $X_{i}$ are also symmetric. Let $\varepsilon=\left(\varepsilon_{i}\right)_{i=1}^{n}$ be a Bernoulli sequence independent of $\left(X_{i}\right)_{i=1}^{n}$. Weak and strong moments of $\left(\varepsilon_{i}\right)_{i=1}^{n}$ are comparable

$$
\left(\mathbb{E} \sup _{s \in S}\left|\sum_{i=1}^{n} s_{i} \varepsilon_{i}\right|^{p}\right)^{1 / p} \leq C\left[\mathbb{E} \sup _{s \in S}\left|\sum_{i=1}^{n} s_{i} \varepsilon_{i}\right|+\sup _{s \in S}\left(\mathbb{E}\left|\sum_{i=1}^{n} s_{i} \varepsilon_{i}\right|^{p}\right)^{1 / p}\right] .
$$

Hence the symmetry of $X_{i}$ yields

$$
\begin{aligned}
& \left(\mathbb{E} \sup _{t \in T}\left|\sum_{i=1}^{n} t_{i} X_{i}\right|^{p}\right)^{1 / p}=\left(\mathbb{E}_{X} \mathbb{E}_{\varepsilon} \sup _{t \in T}\left|\sum_{i=1}^{n} t_{i} X_{i} \varepsilon_{i}\right|^{p}\right)^{1 / p} \\
& \text { 3.6) } \leq 2 C\left[\left(\mathbb{E}_{X}\left(\mathbb{E}_{\varepsilon} \sup _{t \in T}\left|\sum_{i=1}^{n} t_{i} X_{i} \varepsilon_{i}\right|\right)^{p}\right)^{1 / p}+\left(\mathbb{E}_{X} \sup _{t \in T} \mathbb{E}_{\varepsilon}\left|\sum_{i=1}^{n} t_{i} X_{i} \varepsilon_{i}\right|^{p}\right)^{1 / p}\right],
\end{aligned}
$$

since $(a+b)^{p} \leq 2^{p}\left(a^{p}+b^{p}\right)$.

Since $T$ is symmetric, we have for $x \in \mathbb{R}^{n}$,

$$
\mathbb{E}_{\varepsilon} \sup _{t \in T}\left|\sum_{i=1}^{n} t_{i} x_{i} \varepsilon_{i}\right|=\sup _{t \in T_{1}} \sum_{i=1}^{n} t_{i} x_{i}
$$


where

$$
T_{1}:=\left\{\left(\mathbb{E}_{\varepsilon} s_{i}(\varepsilon) \varepsilon_{i}\right)_{i=1}^{n}: s:\{-1,1\}^{n} \rightarrow T\right\}
$$

is an unconditional subset of $\mathbb{R}^{n}$. Estimate (2.7) applied for $T_{1}$ instead of $T$ yields

$$
\begin{aligned}
&\left(\mathbb{E}_{X}\left(\mathbb{E}_{\varepsilon} \sup _{t \in T}\left|\sum_{i=1}^{n} t_{i} X_{i} \varepsilon_{i}\right|\right)^{p}\right)^{1 / p} \\
& \leq C(\alpha)\left[\mathbb{E}_{X} \mathbb{E}_{\varepsilon} \sup _{t \in T}\left|\sum_{i=1}^{n} t_{i} X_{i} \varepsilon_{i}\right|+\sup _{t \in T_{1}}\left(\mathbb{E}\left|\sum_{i=1} t_{i} X_{i}\right|^{p}\right)^{1 / p}\right] .
\end{aligned}
$$

By the symmetry of $X_{i}$ we have

$$
\mathbb{E}_{X} \mathbb{E}_{\varepsilon} \sup _{t \in T}\left|\sum_{i=1}^{n} t_{i} X_{i} \varepsilon_{i}\right|=\mathbb{E} \sup _{t \in T} \sum_{i=1}^{n} t_{i} X_{i}
$$

Moreover,

$$
T_{1} \subset S(T):=\operatorname{conv}\left\{\left(\eta_{i} t_{i}\right)_{i=1}^{n}: \eta \in\{-1,1\}^{n}, t \in T\right\}
$$

hence

$$
\sup _{t \in T_{1}}\left(\mathbb{E}\left|\sum_{i=1}^{n} t_{i} X_{i}\right|^{p}\right)^{1 / p} \leq \sup _{t \in S(T)}\left(\mathbb{E}\left|\sum_{i=1} t_{i} X_{i}\right|^{p}\right)^{1 / p}=\sup _{t \in T}\left(\mathbb{E}\left|\sum_{i=1}^{n} t_{i} X_{i}\right|^{p}\right)^{1 / p} .
$$

Thus

$$
\begin{aligned}
&\left(\mathbb{E}_{X}\left(\mathbb{E}_{\varepsilon} \sup _{t \in T}\left|\sum_{i=1}^{n} t_{i} X_{i} \varepsilon_{i}\right|\right)^{p}\right)^{1 / p} \\
& \leq C(\alpha)\left[\mathbb{E} \sup _{t \in T}\left|\sum_{i=1}^{n} t_{i} X_{i}\right|+\sup _{t \in T}\left(\mathbb{E}\left|\sum_{i=1} t_{i} X_{i}\right|^{p}\right)^{1 / p}\right] .
\end{aligned}
$$

Let $q=p /(p-1)$ be the Hölder's dual of $p$. For $x \in \mathbb{R}^{n}$ we have

$$
\left(\sup _{t \in T} \mathbb{E}_{\varepsilon}\left|\sum_{i=1}^{n} t_{i} x_{i} \varepsilon_{i}\right|^{p}\right)^{1 / p}=\sup _{t \in T_{2}} \sum_{i=1}^{n} t_{i} x_{i}
$$

where

$$
T_{2}=\left\{\mathbb{E}_{\varepsilon} t h(\varepsilon): t \in T, h:\{-1,1\}^{n} \rightarrow \mathbb{R}, \mathbb{E}_{\varepsilon}|h(\varepsilon)|^{q} \leq 1\right\}
$$

is a unconditional subset of $\mathbb{R}^{n}$. Estimate (2.7) applied for $T_{2}$ instead of $T$ yields

$$
\begin{aligned}
\left(\mathbb{E}_{X} \sup _{t \in T} \mathbb{E}_{\varepsilon} \mid\right. & \left.\left.\sum_{i=1}^{n} t_{i} X_{i} \varepsilon_{i}\right|^{p}\right)^{1 / p} \\
& \leq C(\alpha)\left[\mathbb{E}_{X}\left(\sup _{t \in T} \mathbb{E}_{\varepsilon}\left|\sum_{i=1}^{n} t_{i} X_{i} \varepsilon_{i}\right|^{p}\right)^{1 / p}+\sup _{t \in T_{2}}\left(\mathbb{E}\left|\sum_{i=1}^{n} t_{i} X_{i}\right|^{p}\right)^{1 / p}\right] .
\end{aligned}
$$

Proposition 3.1 and the symmetry of $X_{i}$ gives

$$
\mathbb{E}_{X}\left(\sup _{t \in T} \mathbb{E}_{\varepsilon}\left|\sum_{i=1}^{n} t_{i} X_{i} \varepsilon_{i}\right|^{p}\right)^{1 / p} \leq C\left[\mathbb{E} \sup _{t \in T} \sum_{i=1}^{n} t_{i} X_{i}+\sup _{t \in T}\left(\mathbb{E}\left|\sum_{i=1}^{n} t_{i} X_{i}\right|^{p}\right)^{1 / p}\right] .
$$

Since $T_{2} \subset \operatorname{conv} T$ (recall that we assume the symmetry of $T$ ) we have

$$
\sup _{t \in T_{2}}\left(\mathbb{E}\left|\sum_{i=1}^{n} t_{i} X_{i}\right|^{p}\right)^{1 / p} \leq \sup _{t \in \operatorname{conv} T}\left(\mathbb{E}\left|\sum_{i=1}^{n} t_{i} X_{i}\right|^{p}\right)^{1 / p}=\sup _{t \in T}\left(\mathbb{E}\left|\sum_{i=1}^{n} t_{i} X_{i}\right|^{p}\right)^{1 / p} .
$$


Thus

$$
\begin{aligned}
&\left(\mathbb{E}_{X} \sup _{t \in T} \mathbb{E}_{\varepsilon}\left|\sum_{i=1}^{n} t_{i} X_{i} \varepsilon_{i}\right|^{p}\right)^{1 / p} \\
& \leq C(\alpha)\left[\mathbb{E} \sup _{t \in T} \sum_{i=1}^{n} t_{i} X_{i}+\sup _{t \in T}\left(\mathbb{E}\left|\sum_{i=1}^{n} t_{i} X_{i}\right|^{p}\right)^{1 / p}\right] .
\end{aligned}
$$

Estimate (1.4) follows (for symmetric $X_{i}$ 's) by (3.6)-(3.8)

In the case when the variables $X_{i}$ are centered, but not necessarily symmetric let $\left(X_{1}^{\prime}, \ldots, X_{n}^{\prime}\right)$ be an independent copy of $\left(X_{1}, \ldots, X_{n}\right)$. Then $X_{i}-X_{i}^{\prime}$ are symmetric. The Jensen inequality and the assumption on $X_{i}$ imply that for any $p \geq 2$ we have

$$
\left\|X_{i}-X_{i}^{\prime}\right\|_{2 p} \leq 2\left\|X_{i}\right\|_{2 p} \leq 2 \alpha\left\|X_{i}-\mathbb{E} X_{i}\right\|_{p} \leq 2 \alpha\left\|X_{i}-X_{i}^{\prime}\right\|_{p} .
$$

Therefore, Theorem 1.1 applied to $\left(X_{1}-X_{1}^{\prime}, \ldots, X_{n}-X_{n}^{\prime}\right)$ implies

$$
\begin{aligned}
\left(\mathbb{E} \sup _{t \in T}\left|\sum_{i=1}^{n} t_{i} X_{i}\right|^{p}\right)^{1 / p} & \left(\mathbb{E} \sup _{t \in T}\left|\sum_{i=1}^{n} t_{i}\left(X_{i}-\mathbb{E} X_{i}^{\prime}\right)\right|^{p}\right)^{1 / p} \leq\left(\mathbb{E} \sup _{t \in T}\left|\sum_{i=1}^{n} t_{i}\left(X_{i}-X_{i}^{\prime}\right)\right|^{p}\right)^{1 / p} \\
\leq & C(2 \alpha)\left[\mathbb{E} \sup _{t \in T}\left|\sum_{i=1}^{n} t_{i}\left(X_{i}-X_{i}^{\prime}\right)\right|+\sup _{t \in T}\left(\mathbb{E}\left|\sum_{i=1}^{n} t_{i}\left(X_{i}-X_{i}^{\prime}\right)\right|^{p}\right)^{1 / p}\right] \\
& \leq 2 C(2 \alpha)\left[\mathbb{E} \sup _{t \in T}\left|\sum_{i=1}^{n} t_{i} X_{i}\right|+\sup _{t \in T}\left(\mathbb{E}\left|\sum_{i=1}^{n} t_{i} X_{i}\right|^{p}\right)^{1 / p}\right]
\end{aligned}
$$

what finishes the proof in the general case.

Remark 3.2. It follows by the proof of [9. Theorem 2.3] that if $\left(X_{i}\right)_{i=1}^{n}$ are symmetric, independent and for any $i$ moments of $X_{i}$ grow $\beta$-regularly (i.e. (2.9) holds with $\beta$ instead of 2 ), then the comparison of weak and strong moments of suprema of linear combinations of variables $X_{i}$ holds with a constant $C(\beta)=C \beta^{11}$. Therefore, we may follow the constants in the proofs above to obtain that Theorem 1.1 holds with $C(\alpha)=C^{\log _{2}^{2} \alpha}$.

\section{From COMPARISON OF WEAK AND STRONG MOMENTS TO COMPARISON OF WEAK AND STRONG TAILS}

In this Section we prove Corollary 1.3 and Corollary 1.4. To this end we need the following lemma.

Lemma 4.1. Assume $X_{1}, X_{2}, \ldots$ satisfy the assumptions of Theorem 1.1. Then for any $t \in \mathbb{R}^{n}$,

$$
\left\|\sum_{i=1}^{n} t_{i} X_{i}\right\|_{p} \leq C(\alpha)\left(\frac{p}{q}\right)^{\max \left\{1 / 2, \log _{2} \alpha\right\}}\left\|\sum_{i=1}^{n} t_{i} X_{i}\right\|_{q} \quad \text { for } p \geq q \geq 2 .
$$

Proof. Let $\beta:=\max \left\{1 / 2, \log _{2} \alpha\right\}$. It is enough to show that for positive integers $k \leq l$ we have

$$
\left\|\sum_{i=1}^{n} t_{i} X_{i}\right\|_{2 k} \leq C \alpha\left(\frac{k}{l}\right)^{\beta}\left\|\sum_{i=1}^{n} t_{i} X_{i}\right\|_{2 l} .
$$

A standard symmetrization argument shows that we may assume that the random variables $X_{i}$ are symmetric (see the proof of Theorem 1.1] in the non-symmetric case). 
Using the hypercontractivity method [5, Section 3.3], it is enough to show that for $1 \leq i \leq n$,

$$
\left\|s+\frac{t}{2 \sqrt{2} e \alpha}\left(\frac{l}{k}\right)^{\beta} X_{i}\right\|_{2 k} \leq\left\|s+t X_{i}\right\|_{2 l} \quad \text { for all } s, t \in \mathbb{R} .
$$

This reduces to the following claim.

Claim. Suppose that $Y$ is a symmetric random variable such that $\|Y\|_{2 p} \leq \alpha\|Y\|_{p}$ for some $\alpha \geq 1$ and every $p \geq 2$. Let $k \geq l$ be positive integers. Then

$$
\|1+\sigma Y\|_{2 k} \leq\|1+Y\|_{2 l}, \quad \text { where } \sigma:=\frac{1}{2 \sqrt{2} e \alpha}\left(\frac{l}{k}\right)^{\beta} \text {. }
$$

To show the claim observe first that

$$
\|Y\|_{q} \leq \alpha\left(\frac{q}{p}\right)^{\log _{2} \alpha}\|Y\|_{p} \leq \alpha\left(\frac{q}{p}\right)^{\beta}\|Y\|_{p} \quad \text { for } q \geq p \geq 2 .
$$

Moreover we have

$$
\begin{aligned}
\mathbb{E}|1+\sigma Y|^{2 k} & =1+\sum_{j=1}^{k}\left(\begin{array}{l}
2 k \\
2 j
\end{array}\right) \mathbb{E}|\sigma Y|^{2 j} \leq 1+\sum_{j=1}^{k}\left(\frac{e k}{j} \sigma\|Y\|_{2 j}\right)^{2 j} \\
& \leq 1+\sup _{1 \leq j \leq k}\left(\frac{\sqrt{2} e k}{j} \sigma\|Y\|_{2 j}\right)^{2 j},
\end{aligned}
$$

so it is enough to show that

$$
1+\left(\frac{k^{1-\beta} l^{\beta}}{2 j \alpha}\|Y\|_{2 j}\right)^{2 j} \leq\|1+Y\|_{2 l}^{2 k} \quad \text { for } j=1,2 \ldots l .
$$

To this end we will use the following deterministic inequality:

$$
(1+u)^{p} \geq\left(1+\frac{p}{q} u\right)^{q} \geq 1+\left(\frac{p}{q} u\right)^{q} \quad \text { for } p \geq q \geq 1 \text { and } u \geq 0,
$$

and a simple lower bound for $\|1+Y\|_{2 l}^{2 l}$ :

$$
\mathbb{E}|1+Y|^{2 l}=1+\sum_{r=1}^{l}\left(\begin{array}{c}
2 l \\
2 r
\end{array}\right) \mathbb{E}|Y|^{2 r} \geq 1+\sum_{r=1}^{l}\left(\frac{l}{r}\|Y\|_{2 r}\right)^{2 r} .
$$

Assume first that $1 \leq j \leq \frac{k}{l}$. Estimate (4.2) applied with $p=2 j$ and $q=2$ yields

$$
\frac{k^{1-\beta} l^{\beta}}{2 j \alpha}\|Y\|_{2 j} \leq \frac{k^{1-\beta} l^{\beta}}{j^{1-\beta}}\|Y\|_{2} \leq \sqrt{\frac{k l}{j}}\|Y\|_{2},
$$

where the last inequality holds since $\beta \geq \frac{1}{2}$ and $k \geq j l$. Inequalities (4.5) and (4.4) (applied with $p=k / l$ and $q=j$ ) yield

$$
\|1+Y\|_{2 l}^{2 k} \geq\left(1+\left(l\|Y\|_{2}\right)^{2}\right)^{k / l} \geq 1+\left(\sqrt{\frac{k l}{j}}\|Y\|_{2}\right)^{2 j}
$$

so (4.3) holds for $j \leq \frac{k}{l}$.

If $j \geq \frac{k}{l}$ we choose $r=\lceil j l / k\rceil$, then $j l \leq k r \leq 2 j l$. Since $1 \leq r \leq l$, the estimate (4.5) gives

$\|1+Y\|_{2 l}^{2 k} \geq\left(1+\left(\frac{l}{r}\|Y\|_{2 r}\right)^{2 r}\right)^{k / l} \geq\left(1+\left(\frac{l}{r}\|Y\|_{2 r}\right)^{2 r}\right)^{j / r} \geq 1+\left(\frac{l}{r}\|Y\|_{2 r}\right)^{2 j}$ 
where to get the last two inequalities we used $k / l \geq j / r$ and $j / r \geq 1$. Applying estimate (4.2) with $2 j$ and $2 r$ instead of $p$ and $q$ we get

$$
\frac{k^{1-\beta} l^{\beta}}{2 j \alpha}\|Y\|_{2 j} \leq \frac{k^{1-\beta} l^{\beta}}{2 j}\left(\frac{j}{r}\right)^{\beta}\|Y\|_{2 r} \leq \frac{k}{2 j}\|Y\|_{2 r} \leq \frac{l}{r}\|Y\|_{2 r},
$$

which completes the proof of the claim in the remaining case.

Proof of Corollary 1.3. Let

$$
S:=\sup _{t \in T}\left|\sum_{i=1}^{n} t_{i} X_{i}\right| .
$$

By the Paley-Zygmund inequality and (4.1) we have for $t \in T$,

$$
\begin{aligned}
\mathbb{P}\left(\left|\sum_{i=1}^{n} t_{i} X_{i}\right| \geq \frac{1}{2}\left\|\sum_{i=1}^{n} t_{i} X_{i}\right\|_{p}\right) & =\mathbb{P}\left(\left|\sum_{i=1}^{n} t_{i} X_{i}\right|^{p} \geq 2^{-p} \mathbb{E}\left|\sum_{i=1}^{n} t_{i} X_{i}\right|^{p}\right) \\
& \geq\left(1-2^{-p}\right)^{2}\left(\frac{\left\|\sum_{i=1}^{n} t_{i} X_{i}\right\|_{p}}{\left\|\sum_{i=1}^{n} t_{i} X_{i}\right\|_{2 p}}\right)^{2 p} \geq e^{-C_{4}(\alpha) p} .
\end{aligned}
$$

In order to show (1.7) we consider 3 cases.

Case 1. $2 u<\sup _{t \in T}\left\|\sum_{i=1}^{n} t_{i} X_{i}\right\|_{2}$. Then by (4.6)

$$
\sup _{t \in T} \mathbb{P}\left(\left|\sum_{i=1}^{n} t_{i} X_{i}\right| \geq u\right) \geq e^{-2 C_{4}(\alpha)}
$$

and (1.7) obviously holds if $C_{2}(\alpha) \geq \exp \left(2 C_{4}(\alpha)\right)$.

Case 2. $\sup _{t \in T}\left\|\sum_{i=1}^{n} t_{i} X_{i}\right\|_{2} \leq 2 u<\sup _{t \in T}\left\|\sum_{i=1}^{n} t_{i} X_{i}\right\|_{\infty}$. Let us then define

$$
p:=\sup \left\{q \geq 2 C_{4}(\alpha): \sup _{t \in T}\left\|\sum_{i=1}^{n} t_{i} X_{i}\right\|_{q / C_{4}(\alpha)} \leq 2 u\right\} .
$$

By (4.6) we have

$$
\sup _{t \in T} \mathbb{P}\left(\left|\sum_{i=1}^{n} t_{i} X_{i}\right| \geq u\right) \geq e^{-p} .
$$

By (4.1) we have $\sup _{t \in T}\left\|\sum_{i=1}^{n} t_{i} X_{i}\right\|_{p} \leq C(\alpha) u$, so by Theorem 1.1 and Chebyshev's inequality we have

$$
\mathbb{P}\left(S \geq C_{1}(\alpha)(\mathbb{E} S+u)\right) \leq \mathbb{P}\left(S \geq e\|S\|_{p}\right) \leq e^{-p}
$$

for $C_{1}(\alpha)$ large enough. Thus (1.7) holds in this case.

Case 3. $u>\sup _{t \in T}\left\|\sum_{i=1}^{n} t_{i} X_{i}\right\|_{\infty}=\|S\|_{\infty}$. Then $\mathbb{P}(S \geq u)=0$ and (1.7) holds for any $C_{1}(\alpha) \geq 1$.

Proof of Corollary 1.4. The result is an immediate consequence of Theorem 1.1. (4.1) and (1.1) used with $q$ instead of $p$.

\section{Comparison of Weak AND STRONG MOMEnTS OF SUPREMa IMPLIES COMPARISON OF MOMENTS $p$ AND $2 p$}

Proof of Theorem 1.2. We will use the assumption (1.5) for $T$ containing all vectors of the standard base of $\mathbb{R}^{n}$ and their negatives, i.e. we will use only the inequality

$$
\left(\mathbb{E} \sup _{1 \leq i \leq n}\left|X_{i}\right|^{p}\right)^{1 / p} \leq L\left[\mathbb{E} \sup _{1 \leq i \leq n}\left|X_{i}\right|+\left\|X_{1}\right\|_{p}\right] .
$$


Fix $p \geq 2$ and let $n:=\left\lfloor(4 L)^{2 p}\right\rfloor+1, A:=n^{1 / p}\left\|X_{1}\right\|_{p}$. If $A \geq\left\|X_{1}\right\|_{2 p}$, then (1.6) holds with $\alpha=(4 L)^{2}+1$. Hence we may and will assume $A \leq\left\|X_{1}\right\|_{2 p}$.

Obviously

$$
\mathbb{P}\left(\sup _{1 \leq i \leq n}\left|X_{i}\right| \geq t\right) \leq \min \left\{1, n \mathbb{P}\left(\left|X_{1}\right| \geq t\right)\right\}
$$

Moreover, if $\mathbb{P}\left(\left|X_{1}\right| \geq t\right) \leq \frac{1}{n}$,

$$
\begin{aligned}
\mathbb{P}\left(\sup _{1 \leq i \leq n}\left|X_{i}\right| \geq t\right) & =1-\mathbb{P}\left(\left|X_{1}\right|<t\right)^{n}=\mathbb{P}\left(\left|X_{1}\right| \geq t\right) \sum_{k=0}^{n-1} \mathbb{P}\left(\left|X_{1}\right|<t\right)^{k} \\
& \geq \mathbb{P}\left(\left|X_{1}\right| \geq t\right) \cdot n\left(1-\frac{1}{n}\right)^{n-1} \geq \frac{n}{3} \mathbb{P}\left(\left|X_{1}\right| \geq t\right) .
\end{aligned}
$$

Since $\mathbb{P}\left(\left|X_{1}\right| \geq A\right) \leq \frac{1}{n}$ (which follows by the Markov inequality) and $A \leq\left\|X_{1}\right\|_{2 p}$, we have

$$
\begin{aligned}
\mathbb{E} \sup _{1 \leq i \leq n}\left|X_{i}\right|^{2 p} & \geq 2 p \int_{A}^{\infty} t^{2 p-1} \mathbb{P}\left(\sup _{1 \leq i \leq n}\left|X_{i}\right| \geq t\right) d t \geq 2 p \int_{A}^{\infty} t^{2 p-1} \frac{n}{3} \mathbb{P}\left(\left|X_{1}\right| \geq t\right) d t \\
& =\frac{n}{3} \mathbb{E}\left(\left|X_{1}\right|^{2 p}-A^{2 p}\right)_{+} \geq \frac{n}{3}\left(\left\|X_{1}\right\|_{2 p}^{2 p}-A^{2 p}\right) \geq \frac{n}{3}\left(\left\|X_{1}\right\|_{2 p}-A\right)^{2 p}
\end{aligned}
$$

and

$$
\begin{aligned}
\mathbb{E} \sup _{1 \leq i \leq n}\left|X_{i}\right| & \leq A+\int_{A}^{\infty} \mathbb{P}\left(\sup _{1 \leq i \leq n}\left|X_{i}\right| \geq t\right) d t \leq A+n \int_{A}^{\infty} \mathbb{P}\left(\left|X_{1}\right| \geq t\right) d t \\
& \leq A+n \mathbb{E}\left(\left|X_{1}\right| \mathbf{1}_{\left\{\left|X_{1}\right| \geq A\right\}}\right) \leq A+n\left\|X_{1}\right\|_{p} \mathbb{P}\left(\left|X_{1}\right| \geq A\right)^{1-\frac{1}{p}} \\
& \leq A+n^{1 / p}\left\|X_{1}\right\|_{p},
\end{aligned}
$$

where in the last inequality we used again the fact that $\mathbb{P}\left(\left|X_{1}\right| \geq A\right) \leq \frac{1}{n}$.

Thus our choice of $n$ and $A$, and (5.1) (applied to $2 p$ instead of $p$ ) imply that

$$
\begin{aligned}
2 L\left\|X_{1}\right\|_{2 p} & \leq \frac{1}{2} n^{\frac{1}{2 p}}\left\|X_{1}\right\|_{2 p} \leq \frac{1}{2} n^{\frac{1}{2 p}} A+\left(\mathbb{E} \sup _{1 \leq i \leq n}\left|X_{i}\right|^{2 p}\right)^{1 /(2 p)} \\
& \leq \frac{1}{2} n^{\frac{1}{2 p}} A+L\left[\mathbb{E} \sup _{1 \leq i \leq n}\left|X_{i}\right|+\left\|X_{1}\right\|_{2 p}\right] \\
& \leq \frac{1}{2} n^{\frac{1}{2 p}} A+L A+L n^{\frac{1}{p}}\left\|X_{1}\right\|_{p}+L\left\|X_{1}\right\|_{2 p} \\
& \leq\left\|X_{1}\right\|_{p}\left(\frac{1}{2}(4 L+1) n^{\frac{1}{p}}+2 L n^{\frac{1}{p}}\right)+L\left\|X_{1}\right\|_{2 p} \\
& \leq\left(4 L+\frac{1}{2}\right)\left((4 L)^{2}+1\right)\left\|X_{1}\right\|_{p}+L\left\|X_{1}\right\|_{2 p} .
\end{aligned}
$$

Thus

$$
\left\|X_{1}\right\|_{2 p} \leq\left(4+\frac{1}{2 L}\right)\left(16 L^{2}+1\right)\left\|X_{1}\right\|_{p}
$$

Remark 5.1. It is clear from the proof above that we may take $\alpha(L)=C L^{2}$ in Theorem 1.2 .

\section{REFERENCES}

1. R. Adamczak, R. Latała, A. E. Litvak, K, Oleszkiewicz, A. Pajor, and N. TomczakJaegermann, A short proof of Paouris' inequality, Canad. Math. Bull. 57 (2014), no. 1, 3-8. MR 3150710

2. S. Brazitikos, A. Giannopoulos, P. Valettas, and B.H. Vritsiou, Geometry of isotropic convex bodies, Mathematical Surveys and Monographs 196, American Mathematical Society, Providence, RI, 2014 
3. S. J. Dilworth and S. J. Montgomery-Smith, The distribution of vector-valued Rademacher series, Ann. Probab. 21 (1993), no. 4, 2046-2052. MR 1245300

4. P. Hitczenko, Domination inequality for martingale transforms of a Rademacher sequence, Israel J. Math. 84 (1993), no. 1-2, 161-178. MR 1244666

5. S. Kwapień and W. A. Woyczyński, Random series and stochastic integrals: single and multiple, Probability and its Applications, Birkhäuser Boston, Inc., Boston, MA, 1992. MR 1167198

6. R. Latała, Tail and moment estimates for sums of independent random vectors with logarithmically concave tails, Studia Math. 118 (1996), no. 3, 301-304. MR 1388035

7. 251-274. MR 3274967

8. R. Latała and M. Strzelecka, Weak and strong moments of $\ell_{r}$-norms of log-concave vectors, Proc. Amer. Math. Soc. 144 (2016), no. 8, 3597-3608. MR 3503729

9. R. Latała and T. Tkocz, A note on suprema of canonical processes based on random variables with regular moments, Electron. J. Probab. 20 (2015), no. 36, 17. MR 3335827

10. R. Latała and J. O. Wojtaszczyk, On the infimum convolution inequality, Studia Math. 189 (2008), no. 2, 147-187. MR 2449135

11. M. Ledoux and M. Talagrand, Probability in Banach spaces, Classics in Mathematics, Springer-Verlag, Berlin, 2011, Isoperimetry and processes, Reprint of the 1991 edition. MR 2814399

12. G. Paouris, Concentration of mass on convex bodies, Geom. Funct. Anal. 16 (2006), no. 5, 1021-1049. MR 2276533

13. M. Talagrand, Regularity of infinitely divisible processes, Ann. Probab. 21 (1993), no. 1, 362-432. MR 1207231

Institute of Mathematics, University of Warsaw, Banacha 2, 02-097 Warsaw, Poland. E-mail address: rlatala@mimuw.edu.pl, martast@mimuw.edu.pl 